\title{
Ionization and Recombination with Electrons: Laboratory Measurements and Observational Consequences
}

\author{
Daniel Wolf Savin \\ Columbia Astrophysics Laboratory, Columbia University, New York, NY 10027, USA
}

\begin{abstract}
.
Reliably interpreting spectra from both photoionized and electron-ionized cosmic plasmas requires an accurate understanding of the ionization balance of the source and the underlying atomic physics which produces the ionization structure. Uncertainties in ionization balance calculations hamper our ability to model cosmic sources and to infer reliably their properties. Of particular importance are accurate rate coefficients for electron impact ionization (EII) and dielectronic recombination (DR). Here I briefly review the astrophysical implications due to current uncertainties in EII and DR and discuss our ongoing laboratory work aimed to remove these uncertainties.
\end{abstract}

Keywords: electron-ion ionization; electron-ion recombination; elementary processes in plasmas PACS: $34.80 . \mathrm{Kw}$; 34.80.Lx; 52.20.-j; 95.30.Dr

\section{INTRODUCTION}

Spectroscopic observations of astrophysical plasmas are used to infer the properties of the cosmos. The aim of laboratory astrophysics is to reduce atomic physics uncertainties to the point where discrepancies between spectral observations and models tells us something about the observed sources and cannot be attributed to errors in atomic data.

As an example of the role atomic data play in astrophysics, relative abundances of cosmic plasmas can be inferred using

$$
\frac{n_{\mathrm{A}}}{n_{\mathrm{H}}} \propto \frac{1}{\frac{n_{q}}{n_{\mathrm{A}}}} \frac{I_{\text {line }}}{\alpha_{\text {line }}}
$$

where $n_{\mathrm{A}}$ is the particle density of element A, $n_{\mathrm{H}}$ the density of $\mathrm{H}, n_{q}$ the density of charge state $q$ for element A, $I_{\text {line }}$ the intensity of a line from ion $\mathrm{A}^{q+}$, and $\alpha_{\text {line }}$ the rate coefficient for producing this line. This equation clearly shows that accurate ionization balance calculations are needed for reliable relative abundance determinations. This in turn requires accurate ionization and recombination data. Our research aims to improve the electron impact ionization (EII) and electron-ion recombination data used for modeling astrophysical plasmas, thereby improving our understanding of the cosmos.

\section{ASTROPHYSICAL PLASMAS}

Cosmic plasmas can be divided into two broad classes: electron-ionized and photoionized. Electron-ionized plasmas are formed in stellar atmospheres, supernova remnants, 
the interstellar medium, galaxies, and clusters of galaxies. In collisional ionization equilibrium (CIE), an ion typically forms at an electron temperature $T_{e} \sim I_{p} / 2$ where $I_{p}$ is the ionization potential of the ion. In these plasmas the ionization balance is determined largely by the balance of EII and high temperature dielectronic recombination (DR).

Photoionized plasmas form in the environs of planetary nebulae, $\mathrm{H}$ II regions, the intergalactic medium (IGM), X-ray binaries (XRBs), and active galactic nuclei (AGNs). Here the ionization is due to photons and the resulting photoelectrons. In photoionization equilibrium (PIE), an ion forms typically at $T_{e} \sim I_{p} / 20$. In photoionized plasmas low temperature DR is the dominant recombination mechanism for many ions.

Equation 1 demonstrates the importance of reliable atomic collision data for both electron-ionized and photoionized plasmas. We note here another example applicable to electron-ionized plasmas. For a given element in CIE we can write

$$
\frac{n_{q+1}}{n_{q}}=\frac{C_{q}}{\alpha_{q+1}}
$$

where $C_{q}$ is the EII rate coefficient of charge state $q$ forming charge state $q+1$ and $\alpha_{q+1}$ the electron-ion recombination rate coefficient of charge state $q+1$ forming charge state $q$. It is readily apparent that predicted line ratios involving charge states $q$ and $q+1$ will be affected by uncertainties in the ionization and recombination data used for modeling.

In the astrophysics community, the most commonly used ionization balance calculations for electron-ionized plasmas are those of Arnaud and Rothenflug [1], Arnaud and Raymond [2], and Mazzotta et al. [3]. Two commonly used codes for modeling photoionized plasmas include those of Ferland et al. (CLOUDY [4]) and Kallman and Bautista (XSTAR [5]). The reader can find more information about the atomic physics relevant to electron-ionized and photoionized plasmas in these publications and the works cited therein.

\section{ELECTRON IMPACT IONIZATION (EII)}

EII occurs when an electron collides with an atom or ion and knocks out one or more electrons, thereby resulting in two or more outgoing electrons. In astrophysics, the most commonly used EII rate coefficients are from the 1985 paper of Arnaud and Rothenflug [1]. These were updated in 1992 for iron by Arnaud and Raymond [2]. Common wisdom in the astrophysics community has held that these data are reliable (e.g., Ref. [6]). Partly as a result there has been no significant attempt to update the EII data base in over 15 years. However, the common widsom is an incorrect perception. Our review of the EII data base has turned up large uncertainties which call into question the reliability of the EII data used for plasma modeling.

Another compilation of recommended EII data from the late 80s is that of Lennon et al. [7]. These data use essentially the same scant set of laboratory measurements and theoretical calculations as do Arnaud and her collaborators. However, as shown in Fig. 1, the two recommended EII rate coefficients can differ from one another by a factor of 2 or more at temperatures relevant for CIE plasmas. We find similar errors in many other astrophysically relevant isoelectronic sequence. 

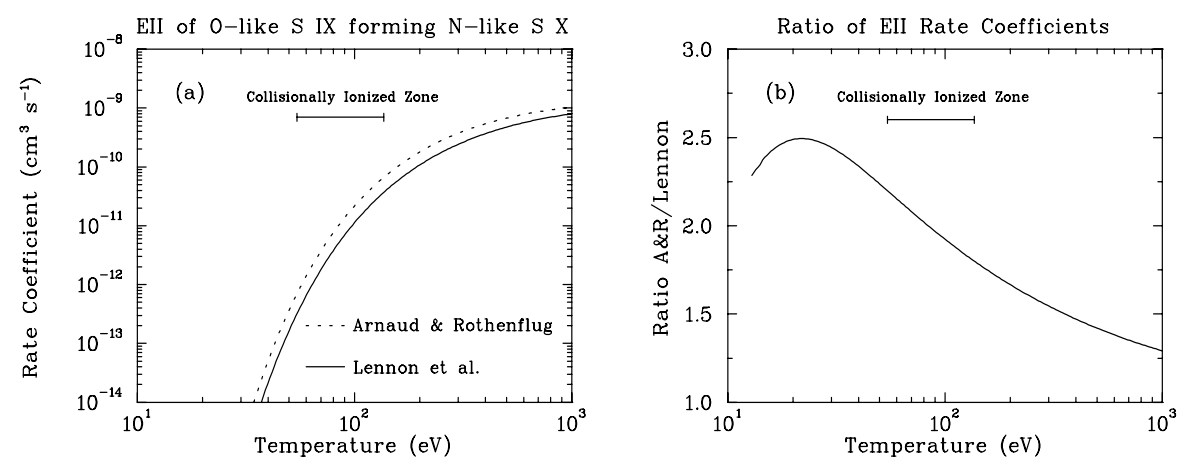

FIGURE 1. Shown for O-like $\mathrm{S}^{8+}$ are (a) the recommended EII rate coefficients of Arnaud \& Rothenflug [1] and Lennon et al. [7] and (b) the ratio of the two. The horizontal bars in (a) and (b) shows the temperature range over which this ion is predicted to form in CIE [3].

EII studies are theoretical and experimentally difficult. Calculations are computationally challenging as they need to take into account an infinite number of final states. Experimental results are often compromised by unknown quantities of metastable state ions in the ion beam. These two difficulties are part of the reason why there has been no significant improvement to the EII data base in the last 15 or so years.

\section{Research Plan}

In order to address the EII data needs of the astrophysics community we have initiated a new series of EII measurements using the Oak Ridge National Laboratory (ORNL) electron-ion crossed-beams apparatus [8]. Recently we have added a gas attenuation cell to the ion beam. This is installed between the ion source and the electron-ion collision region. Using this cell we can determine the metastable fraction of our ion beam. Ground state and metastable state ion can have significantly different cross sections for electron capture (EC) from an attenuating gas. Under these conditions, one of the two states will be preferentially lost as the attenuating gas density is increased. A plot of the logarithm of ion current versus gas density will be bi-linear with the slopes proportional to the EC cross section. Such a plot can be used to infer the relative populations of ground and metastable state ions (e.g., Refs. [9, 10]).

Additionally, we plan on using the attenuation cell to control the metastable fraction of the ion beam. We will carry out EII measurements for two different fractions of metastable ions in the beam $\left(f_{1}\right.$ and $\left.f_{2}\right)$. This will yield as a function of beam energy

$$
\begin{aligned}
& \sigma_{1}(E)=\left(1-f_{1}\right) \sigma_{g}(E)+f_{1} \sigma_{m} \\
& \sigma_{2}(E)=\left(1-f_{2}\right) \sigma_{g}(E)+f_{2} \sigma_{m}
\end{aligned}
$$

where $\sigma_{g}$ and $\sigma_{m}$ are the EII cross sections for the ground and metastable states, respectively. From measurements of $\sigma_{1}, \sigma_{2}, f_{1}$, and $f_{2}$, we will extract the values for $\sigma_{g}$ and 
$\sigma_{m}$. Initial measurements are planned for ions which have long-lived metastable states and for which reliable ground and metastable state EII cross sections measurements have not previously been achieved.

\section{DIELECTRONIC RECOMBINATION (DR)}

DR is a two step recombination process which begins when an electron collisionally excites a core electron of an ion and is simultaneously captured into a Rydberg level. The core electron excitation can be labeled $N l_{j} \rightarrow N^{\prime} l_{j^{\prime}}^{\prime}$ where $N$ is the principal quantum number, $l$ its orbital angular momentum, and $j$ its total angular momentum. The energy of this recombined system lies in the continuum and the complex may autoionize. The DR process is complete when the system emits a photon which reduces the total energy of the recombined system to below its ionization threshold.

The first step of the DR process is commonly called dielectronic capture (DC). Energy conservation requires that the excitation energy $\Delta E$ of the core electron is balanced by the sum of the kinetic energy $E_{k}$ of the incident electron plus the binding energy $E_{b}$ of the level into which it is captured, i.e., $\Delta E=E_{k}+E_{b}$. Because both $\Delta E$ and $E_{b}$ are quantized, $E_{k}$ is also quantized. Hence, DC and hence DR are resonance processes.

Theoretical DR rate coefficients for K-shell ions are believed to be well understood. This is partially due to a sustained series of electron beam ion trap (EBIT) and ion storage ring measurements over the past 15 years. These measurements have provided important benchmarks for the development of modern theoretical methods for calculating DR. Reviews of experimental and theoretical K-shell DR are given in Refs. [11, 12]. Overall agreement between theory and experiment is on the order of $20 \%$.

The situation for L- and M-shell ions, though, has not been so good. DR theory for these many-electron systems are theoretically and computationally challenging and the DR database for these ions has been known to highly unreliable for a number of years. The implications of these DR uncertainties have been explored for solar spectra $[12,13]$, the IGM $[14,15]$, and X-ray photoionized plasmas such as are formed in the environs of XRBs and AGN $[16,17]$.

The importance of reliable M-shell Fe DR data has also been shown by the analyses of recent Chandra and XMM AGN spectra. These spectra show a broad unresolved transition array (UTA) which is attributed to absorption by M-shell Fe ions. Models are able to match AGN spectral features from abundant second row elements. However, these models over-predict the average Fe charge stage. This is believe to be due to the theoretical underestimate of the relevant low temperature DR rate coefficients for Mshell Fe [18, 19].

\section{Research Program}

In order to provide reliable DR data for plasma modeling, we are carrying out storage ring measurements for a wide range of L- and M-shell ions. Our work has focused primarily on DR measurements for iron because it is the highest $Z$ cosmically abundant element and plays an important role in cosmic plasmas in regimes of parameter space 

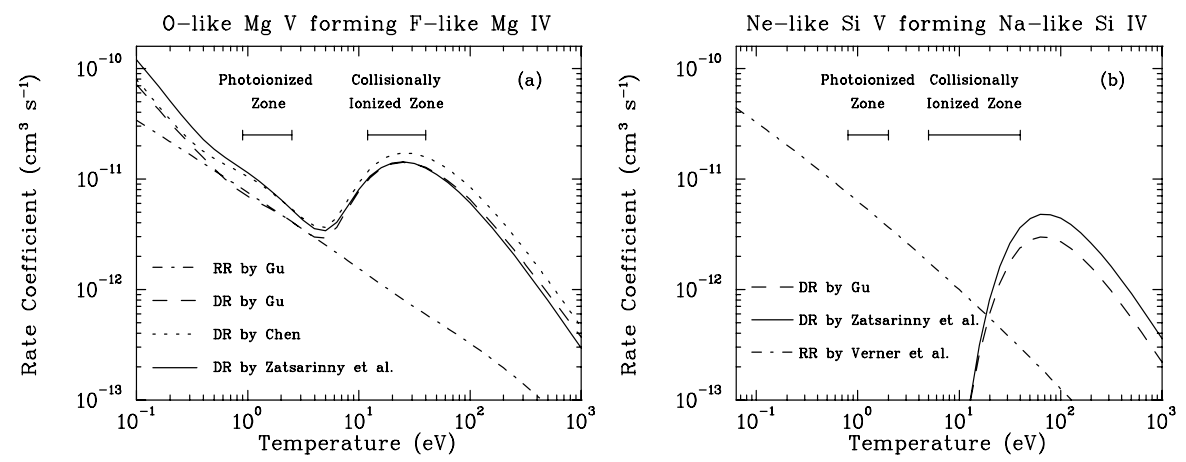

FIGURE 2. Shown are various state-of-the-art DR calculations for (a) O-like $\mathrm{Mg}^{4+}$ forming F-like $\mathrm{Mg}^{3+}$ from Chen [29], Gu [30], and Zatsarinny et al. [31] and (b) Ne-like $\mathrm{Si}^{4+}$ forming Na-like $\mathrm{Si}^{3+}$ from Gu [30] and Zatsarinny et al. [32]. The radiative recombination data in (a) are from Ref. [33] and in (b) from Ref. [34]. The horizontal bars in (a) and (b) shows the temperature range over which each ion is predicted to form in PIE [5] and CIE [3].

where other ions have already been fully stripped. Our work also provides important benchmarks for modern DR theory. These tested methods can then be used to calculated the needed DR data for other ions in the measured isoelectronic sequences. However, as we discuss below, we are finding that even if the various state-of-the-art DR calculations are in good agreement with experiment for a given iron ion, that does not guarantee that they will be in good agreement with one another for isoelectronic ions at lower atomic number $Z$.

We have carried out measurements of DR via $\Delta N=0$ core excitations for Al-like $\mathrm{Fe}^{13+}$ (in preparation), $\mathrm{Mg}$-like $\mathrm{Fe}^{14+}$ (in preparation), $\mathrm{Na}$ like $\mathrm{Fe}^{15+}$ [20, 21], F-like

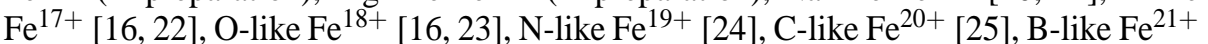
[25], and Be-like $\mathrm{Fe}^{22+}$ (in preparation). We have also carried out $\Delta N=1$ measurements for Na-like $\mathrm{Fe}^{15+}[20,21]$, O-like $\mathrm{Fe}^{18+}[16,23]$, and Be-like $\mathrm{Fe}^{22+}$ (in preparation). Additionally, we note that there have been $\Delta N=0$ and $\Delta N=1 \mathrm{DR}$ measurements for Li-like $\mathrm{Si}^{11+}$ [26] and $\mathrm{Cu}^{26+}$ [27], and also for F-like $\mathrm{Se}^{35+}$ [28]. To summarize, storage ring $\Delta N=0 \mathrm{DR}$ measurements exist for all L-shell ions and a few of the M-shell ions. The situation is far spottier for $\Delta N=1 \mathrm{DR}$ and we are unaware of any storage ring measurements for isoelectronic sequences not mentioned above.

A detailed discussion of typical DR data collection techniques and DR resonance spectra are given in the references above and the papers cited therein. The point which we wish to make here is that even with all the benchmarking that has been going on, for some ions the various state-of-the-art DR calculations have still not converged. This can readily be seen in Fig. 2(a) where the modern DR results differ from one another by a factor of 2.5 at temperatures relevant for photoionized plasmas. This is attributed to the uncertainties in the calculated theoretical energies of the low energy DR resonances which dominate the low temperate DR rate coefficient. Interesting also are the theoretical results for Ne-like ions shown in Fig. 2(b). Though these calculations have yet to be benchmark by storage ring results, one can see that the two published modern DR results differ by a factor of nearly 1.7 . This is surprisingly large considering the typical $20 \%$ 
estimates for the uncertainties in theoretical $\Delta N=1 \mathrm{DR}$ rate coefficients. This large difference is likely due to one of the calculations not having included enough states.

\section{CONCLUSIONS}

Our review of EII has turned up surprisingly large uncertainties in the EII data being used for plasma modeling. Though some of these issues were first noted by Kato et al. [35], these results did not filter into the astrophysics community. Clearly much theoretical and experimental work needs to be done to improve the EII data base. Our laboratory work will help to address this issue.

The situation is somewhat better for DR of L-shell ions. There has been a significant improvement to the DR data base for these ions thanks to a combined experimental and theoretical efforts over the past few years. However, a number of issues remain to be resolved and further experimental work is needed.

For high temperature DR, there is in general good agreement between theory and experiment for the few L-shell isoelectronic sequences which have been measured. But there are some surprising discrepancies between various modern DR calculations for systems which have not yet been benchmarked.

For low temperature DR, state-of-the-art theoretical methods are often still not able to calculate reliable DR rate coefficients. This can be traced directly back to the accuracy of the atomic structure codes used to calculate the energies of the low-lying doubly-excited autoionizing states that are important for low temperature DR. For many-electron ions with partially-filled shells, there has been no significant advance in atomic structure methods for over 30 years. This stands as possibly the greatest challenge for producing reliable low temperature DR rate coefficients. Given the limited laboratory resources which can be devoted to this problem, it will not be possible to measure all the astrophysically relevant low energy DR resonances. Theoretical calculations must be used for all the unmeasured systems. But this cannot be achieved without any advances in atomic structure methods.

The situation is probably even worse for M-shell ions. Few benchmark measurements exist to guide the theory. And any theoretical and computational challenges experienced for L-shell ions will be even greater for M-shell ions.

It is probably fair to summarize this overview by saying that it will take many more years of combined theoretical and experimental efforts before the EII and DR data bases are truly reliable.

\section{ACKNOWLEDGMENTS}

The author thanks his collaborators M. Bannister, E. Landi, J. M. Laming, W. Mitthumsiri, A. Müller, M. Schnell, S. Schippers, and A. Wolf for many stimulating discussions. We also thank T. Kato for providing the EII fit parameters of Lennon et al. in electronic form and for M. Chen for providing his corrected DR fit parameters for $\mathrm{Mg}^{4+}$. DWS supported in part by the NASA Space Astrophysics Research and Analysis program and the NASA Solar Physics Research, Analysis, and Suborbital program. 


\section{REFERENCES}

1. M. Arnaud and R. Rothenflug, Astron. Astrophys. Suppl. Ser. 60, 425-457 (1985).

2. M. Arnaud and J. Raymond, Astrophys. J. 398, 394-406 (1992).

3. P. Mazzotta, G. Mazzitelli, S. Colafrancesco, and N. Vittorio, Astron. Astrophys. Suppl. Ser. 133, $403-$ 409 (1998).

4. G. J. Ferland, K. T. Korista, D. A. Verner, J. W. Ferguson, J. B. Kingdon, and E. M. Verner, Publ. Astron. Soc. Pacific 110, 761-778 (1998).

5. T. Kallman and M. Bautista, Astrophys. J. Suppl. Ser. 133, 221-253 (2001).

6. J. C. Raymond, "Radiation from Hot, Thin Plasma," in Hot Thin Plasmas in Astrophysics, edited by R. Pallavicini, Kluwer Academic Publishers, Dordrecht, 1988, pp. 3-20.

7. M. A. Lennon, K. L. Bell, H. B. Gilbody, J. G. Hughes, A. E. Kingston, M. J. Murray, and F. J. Smith, J. Chem. Phys. Ref. Data 17, 1285-1363 (1988).

8. M. E. Bannister, Phys. Rev. A 54, 1435-1444 (1996).

9. M. Zuo, S. J. Smith, A. Chutjian, I. D. Williams, S. S. Tayal, and B. M. McLaughlin, Astrophys. J. 440, 421-429 (1995).

10. D. B. Reisenfeld, L. D. Gardner, P. H. Janzen, D. W. Savin, and J. L. Kohl, Phys. Rev. A 60, 1153-1164 (1999).

11. A. Müller, At. Plasma Material Interactions 6, 59-100. (1995).

12. D. W. Savin and J. M. Laming, Astrophys. J. 566, 1166-1177 (2002).

13. N. S. Brickhouse, "EUV Spectroscopy of Stellar Coronae," in Atomic Processes in Plasmas Tenth Topical Conference, edited by A. L. Osterheld and W. H. Goldstein, AIP Conference Proceedings 381, American Institute of Physics, Woodbury, New York, 1996, pp 31-38.

14. D. W. Savin, Astrophys. J. 533, 106-112 (2000).

15. A. Aguirre, J. Schaye, T. S. Kim, T. Theuns, M. Rauch, and W. L. Sargent, Astrophys. J. 602, 38-50 (2004).

16. D. W. Savin, et al., Astrophys. J. Suppl. Ser. 123, 687-702 (1999).

17. D. W. Savin, et al., "Measurements of Low Temperature Dielectronic Recombination in L-shell Iron for Modeling X-ray Photoionized Cosmic Plasmas," in Atomic Processes in Plasmas Twelfth Topical Conference, edited by R. C. Mancini and R. A. Phaneuf, AIP Conference Proceedings 547, American Institute of Physics, Woodbury, New York, 2000, pp. 267-278.

18. H. Netzer, Astrophys. J. 604, 551-555 (2004).

19. S. B. Kraemer, G. J. Ferland, and J. R. Gabel Astrophys. J. 604, 556-561 (2004).

20. J. Linkemann, et al., Nucl. Instrum. Methods B 98, 154-157 (1995).

21. A. Müller, Int. J. Mass Spectrom. 192, 9-22 (1999).

22. D. W. Savin, et al., Astrophys. J. Lett. 489, L115-118 (1997).

23. D. W. Savin, et al., Astrophys. J. 576, 1098-1107 (2002).

24. D. W. Savin, et al., Astrophys. J. Suppl. Ser. 138, 337-370 (2002).

25. D. W. Savin, et al., Astrophys. J. Suppl. Ser. 147, 421-435 (2003).

26. J. Kenntner, et al., Nucl. Instrum. Methods B 98, 142-145 (1995).

27. G. Kilgus, D. Habs, D. Schwalm, A. Wolf, N. R. Badnell, and A. Müller, Phys. Rev. A 46, 5730-5740 (1992).

28. A. Lampert, A. Wolf, D. Habs, G. Kilgus, D. Schwalm, M. S. Pindzola, and N. R. Badnell, Phys. Rev. A 53, 1413-1423 (1996).

29. M. H. Chen, Phys. Rev. A 66, 052715 (2002).

30. M. F. Gu, Astrophys. J. 590, 1131-1140 (2003).

31. O. Zatsarinny, T. W. Gorczyca, K. T. Korista, N. R. Badnell, and D. W. Savin, Astron. Astrophys. 412, 587-595 (2003).

32. O. Zatsarinny, T. W. Gorczyca, K. T. Korista, N. R. Badnell, and D. W. Savin, Astron. Astrophys. 426, 699-705 (2004).

33. M. F. Gu, Astrophys. J. 589, 1085-1088 (2003).

34. D. A. Verner and G. J. Ferland Astrophys. J. Suppl. Ser. 103, 467-473 (1996).

35. T. Kato, K. Masai, and M. Arnaud, "Comparison of Ionization Rate Coefficients of Ions from Hydrogen through Nickel," National Institute for Fusion Science Report, Nagoya, Japan, NIFS-DATA14 (1991). 